\title{
Journal of Surgery
}

\section{Anatomy, Physiology and Mechanism of Cardiac Autonomic Nervous System Mediated Atrial Fibrillation}

\author{
Rao Mingliang, Hou Yuemei* \\ Shanghai Jiaotong University, Shanghai, China
}

"Corresponding author: Hou Yuemei, The 6th People's Hospital (Southern), Shanghai Jiaotong University, Nanfeng Highway 6600, Fengxian District, Shanghai, China. Tel: +862157412277; Email: houyuemei@sina.com

Citation: Mingliang R, Yuemei H (2018) Anatomy, Physiology and Mechanism of Cardiac Autonomic Nervous System Mediated Atrial Fibrillation. J Surg: JSUR-1108. DOI: 10.29011/2575-9760. 001108

Received Date: 12 February, 2018; Accepted Date: 22 February, 2018; Published Date: 01 March, 2018

\begin{abstract}
The mechanism of initiation and maintenance of Atrial Fibrillation (AF) mediated by Cardiac Autonomic Nervous System (CANS) has been gained more attention. This article will review the progress in both anatomical structure and physiological regulation mechanism of CANS on cardiac electrical activity, put forward some questions to be solved some thinking as well for discussion.
\end{abstract}

Keywords: Anatomy; Atrial Fibrillation; Cardiac Autonomic Nervous System; Cardiac Electrophysiology

\section{The Anatomy of Cardiac Autonomic Nervous System}

The Autonomic Nervous System (ANS) can be regarded as the interaction between the central nervous system and the viscera, glands, as well blood vessels, which consists of three components: sympathetic, parasympathetic and enteric. Meanwhile, in the ANS (namely vegetative nervous system: VNS), cardiac autonomic nervous system has a more complicated anatomical and physiological functions than other visceral nervous system. The relationship between CANS and AF had been demonstrated since early in 1921 by Lewis and in 1955 by Hoff. While in 1973 R. Lazzara et al, in 1994 W.C. Randall et al and in 1994 J.L. Ardell et al gave rise to the concept of Intrinsic Cardiac Autonomic Nervous System (ICANS), which is made up of Ganglionated Plexi (GP) on the heart itself and on some of the large vessles close to the heart [1].

It can be deduced that CANS is divided into the autonomic innervation to the heart from the brain and the spinal cord (extrinsic autonomic nervous system: ECANS) and the GP on the heart itself comprising the local autonomic nervous systems (ICANS). The ECANS mainly consists of ganglia and their axons located outside the heart. The neurons in the inter mediolateral columns of gray matter of the spinal cord, the stellate ganglia(namely cervicothoracic ganglion, which is merged by inferior cervical ganglion and the first thoracic ganglion, receiving neural inputs mainly from spinal nerves C6-T1), and their axons in route to the heart make up most of the sympathetic limb of ECANS, whereas the solitary tract nucleus, the dorsal vagal nucleus and the vagus nerves superior to GPs constitute most of the parasympathetic limb of ECANS.

The ICANS mainly conclude the GPs embedded in the epicardial Fat Pads (FPs), containing up to several hundred autonomic neurons. There are many animal models for researching the relationship between CANS and cardiac electrophysiology, i.e.: mouse, guinea pig, rabbit, canine, goat, swine, monkey etc, whereas canine has been the main mammalian model to study the influence of ICANS on electrical and mechanical behavior of the heart as well as its clinical significance. Pauza DH et al has been [2-5] researching on human and canine's ICANS morphology and distribution by acetylcholinesterase histochemical staining, which is based on the CANS anatomy and physiological research of Randall WC, Ardell LJ, Armour JA, Chiou C-W, B.J. Scherlag et al, they revealed some of the structural and quantitative differences between human and canine epicardial GPs despite the existing similarity on anatomy.

\section{Structural Differences of CANS Between the Human and Canine}

It is well known that the structural differences between canine's and human's ICANS depend on the sizes of intrinsic cardiac ganglia, which is mainly focus on the epicardial GPs. The ganglia of ICANS were clearly visible in the total heart 


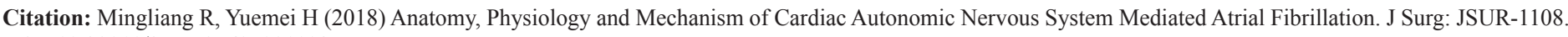
DOI: $10.29011 / 2575-9760.001108$

preparations that has been prepared by Acetylcholinesterase (AchE) histochemical staining. Pauza DH et al. [2,3] have observed that canine and human's cardiac ganglia are mainly cardiac specific globular ganglia and plain ganglia employing contact microscope, counting of neurons distributed inside the globular ganglia using a technique of serial section. Usually, the plain ganglia were small in area and involved no more than 50 neuron somata [4]. In addition, Intramural ganglia were counted using dissecting stereoscopic microscope. The size difference between canine and human intrinsic cardiac ganglia is obvious: the ganglia in human GPs mostly occupied an area less than $0.25 \mathrm{~mm}^{2}$ [4] within $93 \pm 16$ neurons [3] in it on average, whereas the ganglia in canine GPs is divided into three types: small (a few neurons to as large as 0.25 $\mathrm{mm}^{2}$ ), medium (larger than $0.25 \mathrm{~mm}^{2}$ and smaller than $1 \mathrm{~mm}^{2}$ ) and large (larger than $1 \mathrm{~mm}^{2}$ ) sized one within 103,377,945 neurons in them respectively. The biggest canine ganglia have 2000 neurons in $1 \mathrm{~mm}^{2}$. It is obvious that ganglia distinct in size contain a significantly different number of neurons. The GP on canine heart base beneath both pulmonary arteries (GP proximity to SVC-Ao FP or the third FP) presumably from the middle dorsal and dorsal right atrial sub-plexuses, but very rare ganglia were identified on the heart base of human. (Pauza DH's group [3,5] divided intrinsic nerves from locations between ascending aorta and pulmonary trunk, around the pulmonary veins and on every side of superior vena cave extended within epicardium by seven sub-plexuses: left and right coronary sub-plexus, left and middle dorsal sub-plexus, ventral right atrial sub-plexus, ventral left atrial plexus and dorsal right atrial sub-plexus). Besides, the dorsal wall of the ventrical in humans was supplied by nerves derived from left and middle dorsal sub-plexus and left coronary sub-plexus, whereas the same heart region of canine is taken up by nerves of left dorsal subplexus only.

\section{Quantitative Differences of CANS Between the Human and Canine}

Pauza DH's group [3-5] revealed that both ventral and dorsal right atrial sub-plexus are most different between canines and humans quantitatively: these, sub-plexus in canines involve about $70 \%$ of all intrinsic ganglia in each heart while the same sub-plexus was less than $40 \%$ in humans. The two atrial sub-plexuses mainly supply nerves for SAN innervation (SAN-GP, ARGP or the first FP) [2-3]. In order to maintain hemodynamic hemostasis, the atria receive much greater cholinergic innervation than the ventricles and contain more acetylcholine, choline acetyltransferase, and cholinesterase. Secondly, the left and middle dorsal sub-plexus in canines contain about $23 \%$ intrinsic ganglia, whereas they contain more than $50 \%$ intrinsic ganglia in humans. And the neurons quantity within them would be more impressive different: 58000 vs. 28000 neurons on average in canines and humans respectively. On the other hand, left and right dorsal sub-plexus supply nerves mainly to AVN (AVN-GP, IRGP or the second FP). The difference between the canine and human's SAN and AVN nerves innervation would lead to differences of ICANS in cardiac electrophysiology modulation, which is so important in heart rate, atrial and ventricular refractoriness and ventricular contractile force control, and further affect the hemodynamic regulation. Besides above, both the distribution and sizes of the canine epicardiac ganglia were to a great extent depend on the age of animals, reminding that age factor should be controlled in experimental animal's inclusion criteria. Hence, the CANS anatomical difference between canines (as a classical experimental animal model for AF) and human being should be take into consideration so as to better transform basic research into clinical practice.

\section{Questions}

i. At present, CANS's anatomical data obtained through electrophysiological or histological heart studies were far from complete, increasing the uncertainty of targeting and quantizing CANS.

ii. Despite the fact that Pauza DH's group has observed the relatively total ICANS in the nonsectioned canine heart with the aid of dissecting stereomicroscope using AchE histochemistry(Pauza 1992; Pauza et al 1999), cardiac specific globular ganglia were densely packed one above another and their perikarya were almost indiscernible to observers [4] and the neurons deeply inside of globular ganglia were usually unstained as some of reasons lead to the difficulty to reveal ICANS's relative spatial position from integral level and the ECANS's spatial position is more difficult to reveal, forming the gap between CANS's anatomy and physiology.

iii. Excessive reliance on basic experiment conclusion is one of the reasons resulting slow progress of neurogenic AF therapy. ECANS and ICANS in animal models and the human's exhibit species-related differences that may be important in extending experimental data obtained from animals and humans. At the same time, the human ICANS's neural pathway, in contrast to animal ones, have not been mapped and studied electro-physiologically in detail as has been done with animal models [6,7].

\section{Potential Solutions}

i. Improve dissecting stereomicroscope technique as well as accuracy and completeness of CANS's histochemical staining, especially the neurons deeply inside of globular ganglia, getting more complete ICANS's anatomical data and contribute to the establishment of ICANS's morphological framework.

ii. Pay more attention on non-section heart's CANS study. Such as: combine histochemistry staining withheart's fMRI or micro-PET. 


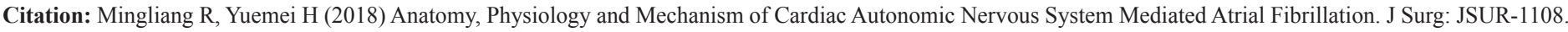
DOI: $10.29011 / 2575-9760.001108$

iii. Study the CANS anatomical differences systematically (include age-related and species-dependent differences), in order to better use CANS data obtained from neural or GP's mapping and other electrophysiology techniques has been done with animal models.

\section{Study on CANS Control Cardiac Electrophysiological Pathway}

ICANS that included GPs serves as more than a relay station for the extrinsic projections of the vagosympathetic system from the brain and spinal cord to the heart, it appears to function interdependently with as well as independently from the ECANS, as evidenced by its retaining nearly full control of cardiac physiology after autotransplantation [1, 8-11].

GPs located in epicardial fat pads and the ligament of Marshall, contain afferent neurons from the atrial myocardium, cholinergic and adrenergic efferent neurons (innervate pulmonary myocardium and atrial myocardium surrounding GP), and interconnecting neurons, which allow communication between GP [9].

Armour et al. put forward "little brain" theory that is closely related with the mammalian animal's cardiac electrophysiological control. "Little brain" theory indicates ICANS retaining nearly full control of cardiac physiology without ECANS $[10,12]$ through three pathways: (1)The variation of regional cardiac mechanosensory or chemosensory milieu $\rightarrow$ afferent neurons $\rightarrow$ efferent sympathetic neurons $\rightarrow$ rebalance of cardiac mechanosensory or chemosensory milieu, (2) The variation of regional cardiac mechanosensory or chemosensory milieu $\rightarrow$ afferent neurons $\rightarrow$ local circuit neurons $\rightarrow$ efferent sympathetic neurons $\rightarrow$ rebalance of cardiac mechanosensory or chemosensory milieu, (3) The variation of regional cardiac mechanosensory or chemosensory milieu $\rightarrow$ afferent neurons $\rightarrow$ local circuit neurons $\rightarrow$ efferent parasympathetic neurons $\rightarrow$ rebalance of cardiac mechanosensory or chemosensory milieu. Of course, the fluctuation of catecholamine cholinergic, peptide and nitrergic substance in blood could be modulated through the ICANS's pathways. Therefore, the interaction that occur among cardiac afferent and efferent neurons are not confined to the central nervous system but also occur within the peripheral autonomic nervous system. And local circuit neurons (neurons that project axons to adjacent neurons as well as those in other ganglia) appears to be responsible for much of the processing of information (namely integration function) within the intrathoracic nervous system, including ICANS [13], which forms the substrate for capacity of the intrathoracic nervous system to display memory. Besides, some of intrinsic cardiac neurons exhibit cardiac cycle-related periodicity that is primarily related to direct cardiac mechano-sensory afferent inputs and, secondarily, to indirect central autonomic efferent inputs [14]. It is the proper range of neurotransmitter's transmission under different space-time between these related afferent, efferent and local ciruit neurons that maintaining the microenvironment of normal operation in each heart region. And their integration and memory function serve as the "little brain" without ECANS (or big brain). However, purely ICANS's modulation course will be confined in the presence of ECANS and it will control cardiac electrophysiology mostly together with ECANS, thus, ICANS becomes important relay station in the course of CANS modulate cardiac electrophysiology. As a result, the cooperation between ECANS and ICANS maintains the microenvironment of cardiac normal operation. B.J. Scherlag, SS Po et al. $[15,16,7]$ have been studying on the CANS's pathways on cardiac electrophysiology. They found some of GPs are the "integration center" between ECANS and ICANS by electrophysiological techniques. Clinical anatomy indicate there are some major epicardial GPs: The Superior Left GP (SLGP) and the Inferior Left GP (ILGP) are located adjacent to the PV-atrial junction of the left superior PV and the left inferior PV, respectively. The anterior right GP (ARGP) is situated at the caudal end of the sinoatrial node, near the right superior PV-atrial junction. And so-called "left atrial neural fold"-Ligament of Marshall (LOM). The Inferior Right GP (IRGP) extends from the inferior right PV-atrial junction to the crux of the heart near the junction of the right atrium and inferior vena cava. Chiou et al. [6] pointed out most efferent vagal fibers to the atria appear to travel through a newly described fat pad located between the medial superior vena cava and aortic root (SVC-Ao fat pad), superior to the right pulmonary artery, and then project onto SAN-FP and AVN-FP and to both atria. Chiou et al. [6] pointed out most efferent vagal fibers to the atria appear to travel through a newly described fat pad located between the medial superior vena cava and aortic root (SVC-Ao fat pad), superior to the right pulmonary artery, and then project onto SAN-FP and AVN-FP and to both atria. Chiou et al. [6] pointed out most efferent vagal fibers to the atria appear to travel through a newly described fat pad located between the medial superior vena cava and aortic root (SVC-Ao fat pad), superior to the right pulmonary artery, and then project onto SAN-FP and AVN-FP and to both atria, which is also previously described by Cooper et al and Mick et al. Thompson proposed SVC-Ao-FP is "the central of cardiac neurons". Butler [17] believe that ARGP plays a important role in contacting left and right GPs. Our team [18] put forward that IVC-LA-FP is the major FP in network controlling AF's initiation and progress.

YL Hou, B.J. Sherlag et al. [16] indicate vagal innervation often travel through multiple GPs before reaching the SA and AV node and GP also modulate the contralateral vagosympathetic inputs. They found some of CANS's neural pathways using autonomic nerve stimulation and GP ablation: (Right Vagosympathetic Trunk (RVG) $\rightarrow$ ARGP $\rightarrow$ SAN) seems to be the main connection between right vagosympathetic trunk and SAN, (left vagosympathetic trunk $\rightarrow \mathrm{SLGP} \rightarrow \mathrm{ARGP} \rightarrow \mathrm{SAN}$ ) seems to 


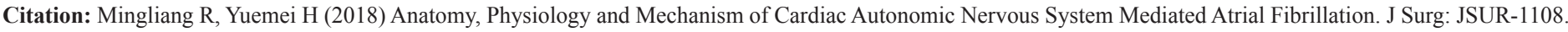
DOI: $10.29011 / 2575-9760.001108$

be the main connection between left vagosympathetic trunk and $\mathrm{SAN} . \quad(\mathrm{RVG} \rightarrow \mathrm{SLGP} \rightarrow \mathrm{ARGP} \rightarrow \mathrm{SAN})$ is also the pathway to SAN. Their data also suggest that ARGP serves as the integration center for both the right and the left vagosympathetic trunks to modulate SR [16], indicative of the role of ARGP as the gateway GP for the sinus node and the presence of other neural pathways bypassing ARGP [8]. In the similar way [16], they elucidated some of the pathways between parasympathetic trunk and AVN: $(\mathrm{LVG} \rightarrow \mathrm{SLGP} \rightarrow \mathrm{ARGP} \rightarrow \mathrm{IRGP} \rightarrow \mathrm{AVN})$ is a major pathway and betweenLVGandAVNand $(\mathrm{LVG} \rightarrow \mathrm{SLGP} \rightarrow \mathrm{IRGP} \rightarrow \mathrm{AVN})$ is another one. (RVG $\rightarrow \mathrm{SLGP} \rightarrow \mathrm{ARGP} \rightarrow \mathrm{IRGP} \rightarrow \mathrm{AVN}$ ) is a major pathway and between RVG and AVN and (RVG $\rightarrow \mathrm{SLGP} \rightarrow \mathrm{IRGP} \rightarrow \mathrm{AVN})$ is another one. These results also indicate that both ARGP and IRGP are the integration centers for both vagosympathetic trunks to innervate the AV node and IRGP is the final converging point and the medium between ARGP and AVN. They also found [15] the effects of anterior right ganglionated plexi on sinus rate slowing were independent of the inferior right ganglionated plexi, but anterior right ganglionated plexi depended on inferior right ganglionated plexi to modulate AV conduction and haven't found the direct functional interconnection between the SLGP and AVN.

\section{Questions}

i. Actually, neurocardiology anatomists and cardiac electrophysiologists have been focusing on the classification and location of intrinsic cardiac ganglia and GPs, which decide the targeting and quantizing CANS's extent after abnormity of CANS induced CANS mediated arrhythmia. However, there is no conclusion on the number of GPs. Although the autonomic ganglia are usually concentrated in several areas covered by epicardial fat pads, the axons and small clusters of autonomic ganglia form an extensive interconnecting neural network, and the crosstalk between ganglia or GPs has far from been understood.

ii. The study on GP's function mainly focus on the first, second $[15,16,18,7]$ and third FP, and only a little study refer to LSGP, LIGP and LOM. Besides, there is no conclusion about "Major Cardiac Region": if there is a or some GPs play a key role in cardiac modulation.

iii. Despite the establishment of "little brain" theory could explain the course of cardiac control, but the pathways between ECANS and ICANS has not been fully revealed and we have not idea about the crosstalk between pathways. Perhaps more complete morphological framework could give birth to more accurate functional framework.

\section{Potential Solutions}

i. Combine the result of neurocardiology anatomy, electrophysiology and clinical anatomy about GPs to ensure the location and number of GPs. Pay more attention on the crosstalk between ganglia and GPs (such as: interneurons local circuit neurons).

ii. Design experiment protocols to research the role of LSGP, LIGP and LOM in cardiac electrophysiological modulation. And their interaction with the first, second and third FP. Combine anatomy, immunohistochemistry and multidimension electrophysiological mapping [7] to further reveal the role of GPs in cardiac control.

iii. Seek the method to combine the pathways in "little brain" and the ones founded through electrophysiological technique, then, complete and detailing the functional framework with the help of morphological framework, after that, we could focus on the crosstalk between pathways (or neurons, ganglias) and establish the network control of cardiac physiology.

\section{The Initiation and Maintenance Mechanism of CANS Mediated AF}

$\mathrm{AF}$ is a common multi-faceted tachyarrhythmia in clinical practice and medical scientists have been tracing it for a long time [19]. Despite the prevailing view of the mechanism for AF was multiple reentrant circuits, the role of CANS mediated AF has obtained increasingly attention in the fields. Moe et al successfully made PAF model induced by abnormal vagus nerve tension in 1959. And Coumel et al reported a group of vagus nerve neurogenic AF cases in 1978 (without organic heart diseases and sinus rate slowing induced secondary AF at night). In the year of 1982, Allessie et al made persistent AF model via the vagus nerve stimulation and synchronous atrial pacing resulting atrial and nerve remodeling, and they proposed the important role of abnormal CANS tension namely atrial nerve remodeling in the course of AF initiation and development. Wijfelis et al. [20] made PerAF through continuous right atrial pacing and put forward the theory of "AF Begets AF" in 1995, indicating pacing induce atrial electrical remodeling is the major mechanism of AF initiation and development. Chiou et al. research [6] indicated the SVC-Ao fat pad receives most of the vagal fibers to the atria and sinus and AV nodes. Elimination of the fat pad with RFCA (radio frequency catheter ablation) selectively vagally denervated the atria and sinus and $\mathrm{AV}$ nodes and inhibit the neurogenic atrial arrhythmia. But the parasympathetic denervation super-sensitivity becomes a difficulty both in basic and clinical study. They proposed that an atrium that is more homogeneous electrically would have a reduced propensity to fibrillate. Besides above, some study suggest that sports and emotion induced sympathetic nerve tension increasing could also trigger paroxysmal atrial fibrillation. Jayachandran et al made AF model via rapid atrial stimulation induced augment on homogeneity of atrial sympathetic nerve innervation and sympathetic activity in 2000 . In essence, the distribution homogeneity of intrinsic cardiac nerve 


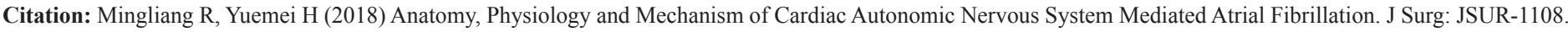
DOI: $10.29011 / 2575-9760.001108$

and nerves denervation super-sensitivity are both part of imbalance between sympathetic and parasympathetic nerve, but they have different extent of imbalance, which both could tend to rebalance through nerves modulation and it is helpful to the termination of neurogenic AF. After that, Pachon et al proposed atrial structural remodeling is a important mechanism of AF initiation and progress in 2004. Patterson et al. [21] study the relation between CANS and AF through high-frequency electrical stimulate pulmonary vein specimen in vitro triggered AF, proposing the theory of "CalciumTriggering”. Then, J Zhou, B.J Sherlag et al. [7] proposed the "Octopus" hypothesis: A hyperactive state of the GP (head of octopus) may trigger local release of a gradient of excessive amounts of neurotransmitters and subsequently initiate AF. When the axons (tentacles) are excited, the GP can be retrogradely activated and elicit a similar response. B.J. Sherlag et al. [22] suggest that RAP not only induces atrial electrical remodeling but also promotes autonomic remodeling. These two remodeling processes may form a vicious cycle and each may perpetuate other, which help to explain the mechanism of AF maintains itself in its very early stage. Nakagawa. $H$. et al. [9] found that stimulation of the GP produces both parasympathetic stimulation (markedly shortens action potential duration) and sympathetic stimulation (increases calcium transient) in the pulmonary vein myocardium and atrial myocardium. Chen P S et al. [23,24] simultaneously recording the sympathetic and parasympathetic tension during AF and showed that both sympathetic and parasympathetic nerves take part in the initiation and maintenance of AF, they verified that both sympathetic and parasympathetic nerves have different extent of opposite element employing immunohistochemistry and proposed the potential benefit and limitations of neuromodulation in the management of AF.

\section{Questions}

i. There is only a little study on purely CANS mediated AF, perhaps because the nerves are tightly adjacent to other tissues and the complexity of CANS modulation, leading to a difficulty on assessing the upstream role of CANS during AF's initiation and maintenance.

ii. The hypothesis and theories have been proposed could only explain some of phenomena about CANS mediated AF, the deficiency of systematicness about CANS study has to be take into consideration.

iii. What relationship between rapid atrial pacing induced atrial electrical remodeling, nerve remodeling and structural remodeling? Are, they take place simultaneously or sequentially or a part of sequentially and the rest simultaneously? What is the trigger or marker of formation of vicious cycle between three remodeling processes and the major step(s) for AF substrate formation? What is the mechanism of AF after the first few hours of AF and the PAF change into PerAF? How to break the vicious cycle with least tissue damagement so as to alleviate nerves denervation super-sensitivity and reduce the reccurence rate?

\section{Potential Solutions}

i. Make purely neurogenic animal AF model and study definite role of CANS in neurogenic AF and its proportion in the mechanism of $\mathrm{AF}$ initiation and maintenance, laying the foundation of clinical intervention for neurogenic AF.

ii. Combine the morphological and functional framework of CANS, seek the protocol to study the relationship between CANS and AF in situ. Expand the clinical cases and extend the time of follow-up.

\section{Study on The CANS Intervention for The Management of AF}

$\mathrm{T} \mathrm{he}$

relationship between autonomic nervous derived from the brain and AF was well established during last century [1]. The management of CANS related AF have experienced the course of medicine, direct current cardioversion, surgical MAZE procedure, radio frequency catheter ablation, GPs ablation, renal sympathetic denervation $[22,25]$, cervical vagal nerve stimulation, baroreflex stimulation, cutaneous stimulation [26, 27-31], acupuncture (such as, neiguan) [24] and biological therapies. Novel drug approaches develop slowly, and it has the risk of proarrhythmia. Clinical electrophysiologists discovered that the majority of patients with PAF, resistant to drugs and cardioversion, had focal, ectopic firing arising from the myocardial sleeves covering the PVs and developed CVPI which would supposedly isolate the PVs from atria [32]. After that, some practitioners found that CPVI combined GP ablation could enhance the successful rate of PAF cases [33]. However, the obvious low successful rate on PerAF cases, nerves denervation super-sensitivity, all kinds of ablation methods have no obvious advantages, low postoperative sinus rate maintenance rate for PAF and PerAF cases have limit the expend of ablation in the fields of AF therapy. B.J. Sherlag, SS. Po et al. [34-39, 26] suggested Low Level Vagus Nerve Stimulation (LL-VNS) or Low Level Transcutaneous Electrical Stimulation (LL-TS) can reverse RAP-induced atrial remodeling, inhibit AF inducibility and cholinergic nerve stimulation induced AF. And LL-TS is a potential noninvasive treatment of AF. Low Level Superior Vena Cave Stimulation (LL-SVCS) without nerve exposure and LL-TS etc. neuromodulation methods can potentially break the vicious cycle of "AF begets $A F$ ", reverse remodeling and reduce $\mathrm{AF}$ inducibility [26,40]. Chen P S et al. [41] indicated that LL-VNS can inhibit activity of stellate ganglion and reduce the occurrence of paroxysmal atrial arrhythmia. Armour et al. [14] believed that modulation of stochastic intrinsic cardiac local circuit neural recruitment may represent a novel target for the treatment of select 
Citation: Mingliang R, Yuemei H (2018) Anatomy, Physiology and Mechanism of Cardiac Autonomic Nervous System Mediated Atrial Fibrillation. J Surg: JSUR-1108. DOI: $10.29011 / 2575-9760.001108$

cardiac disease, including atrial arrhythmias.

\section{Questions}

i. Despite plenty of study about the relationship between CANS and $\mathrm{AF}$, the terminal point of clinical $\mathrm{AF}$ therapy will be a puzzle without establishment of sympathetic and parasympathetic nerve tension's age-related and species-dependent physiology and rebalance scope.

ii. The mechanism of RSD treat AF simultaneously with drugresistenthypertensionhasfarfrombeenfullyunderstood[22,25].

iii. The uncertainty of GPs role in cardiac electrophysiology control limit the status of autonomic nerves denervation in ablation and the further expend of AF ablation.

\section{Potential Solutions}

i. Study sympathetic and parasympathetic nerve tension's scope from the view of physiology and pathology with the help of neuromuscular electrical diagram so as to obtain the fluctuation range of CANS rebalance during pathological condition, laying the foundation for clinical neuromodulation.

ii. Make animal model of AF simultaneously with drug-resistent hypertension, further reveal the role of RSD in it and obtain enlightment from it about CANS mediated AF.

iii. Further reveal the role of GPs in cardiac electrophysiology, reducing nerves lesion during ablation and alleviate the phenomena of nerves denervation super-sensitivity.

\section{Summarize}

In sharp contrast to intracardiac neurons, intrinsic neurons in the esophagus, stomach or guts are obviously more widely distributed along the entire alimentary canal and, these nerve cells may form comparatively smaller intramural ganglia containing no more than 20-100 neurons per ganglion [42]. The neurons located inside of intramural cardiac ganglia are frequently globular with densely packed neurons. The globular ganglia should be considered as characteristic for cardiac tissue because such ganglia have not been demonstrated in other visceral organs. In spite of evident species-dependent differences with respect to frequency of large ganglia, the majority of intrinsic cardiac ganglia both in animals and humans were small in area ranging from 0.01 to $0.17 \mathrm{~mm}^{2}$ [4]. Both the complexity and large size of some cardiac ganglia are presumably associated with a condensed distribution of intrinsic cardiac neurons almost without exception on the atria, and innervate different region of heart through axons. Besides, there are different extent of overlapping between neurons inside ganglias, this type of overlapping make the relation between neurons more closer because of spatial adjacent to each other, leading to more frequently crosstalk and forming many circuits by inter-neurons and local circuit neurons $[13,14]$. Perhaps it is these local neurons circuits $[13,14]$ play a key role in the course of ICANS control cardiac electrophysiology. Therefore, the complexity of intrinsic cardiac ganglia's morphology implies its complexity of function. Horack guinea et al. found that nearly $90 \%$ of all identified neurons in the atria of the guinea pig were located in ganglia(ganglionic neurons) and only $10 \%$ of all neurons loosely distributed outside of ganglia(individual neurons), which indicates the relationship between the number of neurons located inside of intramural cardiac ganglia and its area may be a tool for approximation of neurons number that are intrinsically involved in heart control, which is a enlightment for targeting and quantizing GPs or noninvasively neural tension's modulation to build new ambulatory balance between sympathetic and parasympathetic tension. However, there are many gaps between CANS anatomy, physiology, and neurogenic mechanism of $\mathrm{AF}$, which require further basic and clinical research.

\section{References}

1. He B, Scherlag BJ, Nakagawa H, Lazzara R, Po SS (2012) The Intrinsic Autonomic Nervous System in Atrial Fibrillation: A Review. ISRN Cardiology 2012: p. 1-8.

2. Pauza DH, Skripka V, Pauziene N, Stropus R (1999) Anatomical study of the neural ganglionated plexus in the canine right atrium: implications for selective denervation and electrophysiology of the sinoatrial node in dog. Anat Rec 255: p. 271-294.

3. Pauza DH, Skripka V, Pauziene N, Stropus R (2000) Morphology, distribution, and variability of the epicardiac neural ganglionated subplexuses in the human heart. Anat Rec 259: p 353-82.

4. Pauza DH, Pauziene N, Pakeltyte G, Stropus R (2002) Comparative quantitative study of the intrinsic cardiac ganglia and neurons in the rat, guinea pig, dog and human as revealed by histochemical staining for acetylcholinesterase. Ann Anat 184: p. 125-36.

5. Pauza DH, Skripka V, Pauziene N (2002) Morphology of the Intrinsic Cardiac Nervous System in the Dog: A Whole-Mount Study Employing Histochemical Staining with Acetylcholinesterase. Cells Tissues Organs 172: p. 297-320.

6. Chiou CW, Eble JN, Zipes DP (1997) Efferent vagal innervation of the canine atria and sinus and atrioventricular nodes. The third fat pad. Circulation 95: p. 2573-2584.

7. Zhou J, Scherlag BJ, Edwards J, Jackman WM, Lazzara R, et al. (2007) Gradients of Atrial Refractoriness and Inducibility of Atrial Fibrillation due to Stimulation of Ganglionated Plexi. Journal of Cardiovascular Electrophysiology 18: p 83-90.

8. Sun J, et al. (2014) "Role of the Autonomic Nervous System in Atrial Fibrillation." Cardiac Electrophysiology:From Cell to Bedside 2014: p. 469-474.

9. Nakagawa H, Scherlag BJ, Patterson E, Ikeda A, Lockwood D, et al. (2009) Pathophysiologic basis of autonomic ganglionated plexus ablation in patients with atrial fibrillation. Heart Rhythm 6: p. S26-S34.

10. Murphy DA, Thompson GW, Ardell JL, McCraty R, Stevenson RS, et al. (2000) The heart reinnervates after transplantation. Ann Thorac Surg 69: p. 1769-1781. 
Citation: Mingliang R, Yuemei H (2018) Anatomy, Physiology and Mechanism of Cardiac Autonomic Nervous System Mediated Atrial Fibrillation. J Surg: JSUR-1108. DOI: $10.29011 / 2575-9760.001108$

11. Armour JA (2008) Potential clinical relevance of the 'little brain' on the mammalian heart. Experimental Physiology 93: p. 165-176.

12. Murphy DA, O’Blenes S, Hanna BD, Armour JA (1994) Capacity of intrinsic cardiac neurons to modify the acutely autotransplanted mammalian heart. J Heart Lung Transplant 13: p. 847-856.

13. Armour JA (2010) Functional anatomy of intrathoracic neurons innervating the atria and ventricles. Heart Rhythm 7: p. 994-996.

14. Beaumont E, Salavatian S, Southerland EM, Vinet A, Jacquemet V, et al. (2013) Network interactions within the canine intrinsic cardiac nervous system: implications for reflex control of regional cardiac function. The Journal of Physiology 591: p 4515-4533.

15. Hou Y, Scherlag BJ, Lin J, Zhou J, Song J, et al. (2007) Interactive atrial neural network: Determining the connections between ganglionated plexi. Heart Rhythm 4: p. 56-63.

16. Hou Y, Scherlag BJ, Lin J, Zhang Y, Lu Z, et al. (2007) Ganglionated Plexi Modulate Extrinsic Cardiac Autonomic Nerve Input. Journal of the American College of Cardiology 50: p. 61-68.

17. Butler CK, Smith FM, Cardinal R, Murphy DA, Hopkins DA, et al. (1990) Cardiac responses to electrical stimulation of discrete loci in canine atrial and ventricular ganglionated plexi. Am J Physiol 259: $p$. H1365-73.

18. Hou Y, Hu J, Po SS, Wang H, Zhang L, et al. (2013) Catheter-Based Renal Sympathetic Denervation Significantly Inhibits Atrial Fibrillation Induced by Electrical Stimulation of the Left Stellate Ganglion and Rapid Atrial Pacing. PLoS ONE 8: p. e78218.

19. Fye WB (2006) Tracing atrial fibrillation--100 years. N Engl J Med 355: p. $1412-1414$.

20. Wijffels MC, Kirchhof CJ, Dorland R, Allessie MA (1995) Atrial fibrillation begets atrial fibrillation. A study in awake chronically instrumented goats. Circulation 92: p. 1954-1968.

21. Patterson E, Po SS, Scherlag BJ, Lazzara R (2005) Triggered firing in pulmonary veins initiated by in vitro autonomic nerve stimulation. Heart Rhythm 2: p. 624-631.

22. Tsai WC and Chen PS (2014) Cross talk between renal and cardiac autonomic nerves: is this how renal denervation works? J Cardiovasc Electrophysiol 25: p. 1257-1258.

23. Tan AY, Zhou S, Ogawa M, Song J, Chu M, et al. (2008) Neural mechanisms of paroxysmal atrial fibrillation and paroxysmal atrial tachycardia in ambulatory canines. Circulation 118: p. 916-925.

24. Chen PS, Chen LS, Fishbein MC, Lin SF, Nattel S (2014) Role of the autonomic nervous system in atrial fibrillation: pathophysiology and therapy. Circ Res 114: p. 1500-1515.

25. Symplicity HTN-2 Investigators, Esler MD, Krum H, Sobotka PA, Schlaich MP, Schmieder RE (2010) Renal sympathetic denervation in patients with treatment-resistant hypertension (The Symplicity HTN-2 Trial): a randomised controlled trial. The Lancet 376: p. 1903-1909.

26. Yu L, Scherlag BJ, Li S, Fan Y, Dyer J, et al. (2013) Low-level transcutaneous electrical stimulation of the auricular branch of the vagus nerve: A noninvasive approach to treat the initial phase of atrial fibrillation. Heart Rhythm 10: p. 428-435.

27. Li S, Zhou X, Yu L, Jiang H (2015) Low level non-invasive vagus nerve stimulation: A novel feasible therapeutic approach for atrial fibrillation. International Journal of Cardiology 182: p. 189-190.
28. Chen M, Yu L, Liu Q, Wang Z, Wang S, et al. (2015) Low level tragus nerve stimulation is a non-invasive approach for anti-atrial fibrillation via preventing the loss of connexins. International Journal of Cardiology 179: p. 144-145.

29. Stavrakis S, Humphrey MB, Scherlag BJ, Hu Y, Jackman WM, et al. (2015) Low-level transcutaneous electrical vagus nerve stimulation suppresses atrial fibrillation. J Am Coll Cardiol 65: p. 867-875.

30. Gao M, Zhang L, Scherlag BJ, Huang B, Stavrakis S, et al. (2015) Low-level vagosympathetic trunk stimulation inhibits atrial fibrillation in a rabbit model of obstructive sleep apnea. Heart Rhythm 12: p. 818824

31. Chen M, Yu L, Liu Q, Wang Z, Wang S, et al. (2015) Noninvasive vagus nerve stimulation: A novel promising modulator for cardiac autonomic nerve system dysfunction. International Journal of Cardiology 187: p. 338-339.

32. Scherlag BJ, Patterson E, Po SS (2006) The neural basis of atrial fibrillation. J Electrocardiol, 2006. 39: p. S180-183.

33. Katritsis DG, Pokushalov E, Romanov A, Giazitzoglou E, Siontis GC, et al. (2013) Autonomic denervation added to pulmonary vein isolation for paroxysmal atrial fibrillation: a randomized clinical trial. J Am Coll Cardiol 62: p. 2318-2325.

34. Li S, Scherlag BJ, Yu L, Sheng X, Zhang Y, et al. (2009) Low-level vagosympathetic stimulation: a paradox and potential new modality for the treatment of focal atrial fibrillation. Circ Arrhythm Electrophysiol 2: p. 645-651.

35. Yu L, Scherlag BJ, Li S, Sheng X, Lu Z, et al. (2011) Low-level vagosympathetic nerve stimulation inhibits atrial fibrillation inducibility: direct evidence by neural recordings from intrinsic cardiac ganglia. $\mathrm{J}$ Cardiovasc Electrophysiol 22: p. 455-463.

36. Sheng X, Scherlag BJ, Yu L, Li S, Ali R, et al. (2011) Prevention and reversal of atrial fibrillation inducibility and autonomic remodeling by low-level vagosympathetic nerve stimulation. J Am Coll Cardiol 57: p. 563-571.

37. Yu L, Dyer JW, Scherlag BJ, Stavrakis S, Sha Y, et al. (2015) The use of low-level electromagnetic fields to suppress atrial fibrillation. Heart Rhythm 12: p. 809-817.

38. Chen M, Yu L, Ouyang F, Liu Q, Wang Z, et al. (2015) The right side or left side of noninvasive transcutaneous vagus nerve stimulation: Based on conventional wisdom or scientific evidence? International Journal of Cardiology 187: p. 44-45.

39. Stavrakis S and Po SS (2015) Neuroimmunomodulation: A new frontier of treating cardiovascular diseases. Trends in Cardiovascular Medicine 2015.

40. Yu L, Scherlag BJ, Sha Y, Li S, Sharma T, et al. (2012) Interactions between atrial electrical remodeling and autonomic remodeling: how to break the vicious cycle. Heart Rhythm 9: p. 804-809.

41. Shen MJ, Shinohara T, Park HW, Frick K, Ice DS, et al. (2011) Continuous low-level vagus nerve stimulation reduces stellate ganglion nerve activity and paroxysmal atrial tachyarrhythmias in ambulatory canines. Circulation 123: p. 2204-2212.

42. Gabella G (1987) The number of neurons in the small intestine of mice, guinea-pigs and sheep. Neuroscience 22: p. 737-752. 